\title{
Relaciones entre neurocognición, procesamiento emocional y funcionamiento social en la esquizofrenia
}

\author{
Paola Jaramillo, Juan Carlos Ruiz e Inma Fuentes* \\ Facultad de Psicología. Universidad de Valencia
}

(Recibido 4 Septiembre 2011; Aceptado 13 Octubre 2011)

\begin{abstract}
RESUMEN: En la esquizofrenia se presentan una serie de déficits cognitivos que han hecho que la investigación y la práctica profesional actual se centren en el estudio de la neurocognición y la cognición social. Estos déficits tienen importantes implicaciones en el funcionamiento social. El objetivo del presente trabajo es analizar la relaciones entre neurocognición, cognición social, evaluada con tareas de reconocimiento de la emoción, y funcionamiento social. Sesenta personas con diagnóstico de esquizofrenia han formado la muestra y las áreas evaluadas han sido: funcionamiento ejecutivo y flexibilidad cognitiva, atención y vigilancia, memoria, velocidad de procesamiento, identificación y discriminación de emociones y funcionamiento comunitario. Los resultados indican que medidas de cognición básica correlacionan de forma significativa con el funcionamiento comunitario, mientras que medidas de reconocimiento de la emoción, específicamente la identificación de emociones faciales solo correlacionó de forma positiva con un área del funcionamiento comunitario, la de comunicación y contacto social.
\end{abstract}

Palabras clave: esquizofrenia, procesamiento emocional, cognición social, neurocognición y funcionamiento social.

Relationships between neurocognition, emotional processing and social functioning in schizophrenia

\begin{abstract}
A series of cognitive deficits in schizophrenia have led to a focus on neuro- and social cognition in current research and professional practice. These deficits have significant implications for social functioning. The aim of the current study is to analyse the relationships between neurocognition, social cognition (evaluated via emotional recognition tasks) and social functioning. Sixty people diagnosed with schizophrenia made up the sample and the following areas were evaluated: executive functioning and cognitive flexibility, attention and vigilance, processing speed, emotion identification and discrimination and community functioning. Results indicate that measures of basic cognition correlate significantly with communal functioning while measures of emotion recognition, especially those identifying facial emotions only correlate positively with one area of communal functioning, namely that of communication and social contact.
\end{abstract}

Key words: schizophrenia, emotional processing, social cognition, neurocognition and social functioning.

*Correspondencia: Inmaculada Fuentes, Departamento de Personalidad, Evaluación y Tratamientos Psicológicos, Facultad de Psicología, Avda.Blasco Ibañez n²1 46010, Valencia. E-mail:inmaculada. 


\section{INTRODUCCIÓN}

La esquizofrenia es un trastorno mental grave caracterizado, entre otros aspectos, por presentar una serie de déficits cognitivos a los que se ha prestado una gran atención en las últimas décadas y que han dado origen a iniciativas como el proyecto de investigación de la medición y del tratamiento para mejorar la cognición en esquizofrenia (Measurement and Treatment Research to Improve Cognition in Schizophrenia, MATRICS) (Barch, 2005). Este proyecto, señala siete déficits cognitivos críticos: velocidad de procesamiento, atención/vigilancia, memoria de trabajo, aprendizaje y memoria verbal, aprendizaje y memoria visual, razonamiento y solución de problemas, y cognición social (Green y Nuechterlein, 2004). Estos déficits se presentan en un alto porcentaje en las personas con esquizofrenia, y en un $40 \%$ de los casos la afectación cognitiva se considera grave (Gold y Harvey, 1993). Una revisión realizada por Rund (1998) de quince estudios longitudinales de evaluación neuropsicológica, concluyó que, desde el inicio de la enfermedad, los déficits cognitivos son relativamente estables en el tiempo. Esta disfunción cognitiva hallada en los primeros años de la enfermedad, según Hoff, Sakuma, Wieneke, Horon, Kushner y De Lisi (1999) tiende a estabilizarse en un nivel de 1 ó 2 desviaciones estándar por debajo del rendimiento de las personas normales.

El impacto de los deficits cognitivos sobre las áreas de funcionamiento social también ha sido objeto de múltiples investigaciones. Una revisión de 39 estudios publicados realizada por Green, Kern, Braff y Mintz (2000), concluyó que diferentes tipos de déficits cognitivos están asociados con diversas áreas del funcionamiento social y que estos déficits cognitivos podrían de forma separada predecir el resultado funcional. En general, los hallazgos sugieren que la neurocognición explica entre un $20 \%$ y un $60 \%$ de la varianza en el rendimiento funcional de personas con esquizofrenia (Green et al., 2000).

Los estudios en cognición básica son numerosos y han descrito durante años, con evidencias sólidas, las características de estos déficits. Sin embargo la cognición social comienza a ser objeto de las investigaciones sólo desde mediados de los años 90. La cognición social hace referencia a las operaciones mentales que subyacen en las interacciones sociales, y que incluyen los procesos involucrados en percibir, interpretar y generar respuestas a las intenciones, disposiciones y conductas de otros (Brothers, 1990). La cognición social incluye varias áreas: el Procesamiento Emocional, la Teoría de la Mente, la Percepción Social, el Conocimiento Social y Esquemas Sociales, y el Estilo Atribucional.

El Procesamiento Emocional es una de las áreas de la que más se ha nutrido el conocimiento empírico de la cognición social. Los déficits en el rendimiento en las tareas de Procesamiento Emocional, en las personas con esquizofrenia, se presentan tanto en la identificación como en la discriminación de emociones al ser comparados con grupos control de personas sin diagnóstico psiquiátrico (Penn, Combs y Mohamed, 2001; Schneider at al., 2006). Estos déficits han sido asociados a las habilidades cognitivas básicas (Bryson, Bell, y Lysaker, 1997; 
Kee, Kern y Green, 1998; Schneider, Gur, Gur, y Shtatsel, 1995) así como también al funcionamiento social (Hooker y Park, 2002; Ihnen, Penn, Corrigan y Martin, 1998; Mueser et al., 1996; Penn, Spaulding, Reed y Sullivan, 1996; Poole, Tobias y Vinogradov, 2000; Sullivan, Marder, Liberman, Donahoe y Mintz, 1990) y al funcionamiento laboral (Kee, Green, Mintz y Brekke, 2003).

En estos momentos existen varios modelos explicativos de la relación entre neucognición, cognición social y funcionamiento social en personas con esquizofrenia. Estos modelos generados a partir de resultados empíricos indican que la cognición social podría funcionar como un mediador o vínculo entre el funcionamiento neucognitivo y el funcionamiento social (Green y Nuechterlein, 1999; Brekke, Kay, Lee y Green, 2005). En esta línea, el modelo de Vauth, Rüsch, Wirtz y Corrigan (2004) muestra resultados del rol de la cognición social y no social (neurocognición) como determinantes de las habilidades sociales relacionadas con el trabajo. En este estudio, una amplia proporción de la cognición social $(83 \%)$ fue explicada por la cognición no social, y a su vez la cognición social tuvo un valor predictivo más fuerte sobre el funcionamiento vocacional que la cognición no social. Un análisis más completo del tema se puede encontrar en Jaramillo, Fuentes y Ruiz (2009).

Sin embargo la diversidad de variables neurocognitivas, de cognición social y de funcionamiento social, manejadas en las distintas investigaciones así como las diferentes pruebas utilizadas para su evaluación, y las distintas características de pacientes, ha hecho que los modelos que se han descrito sean relativamente heterogéneos. En este trabajo se estudia las relaciones entre neurocognición, cognición social y funcionamiento social en pacientes ambulatorios, estables y con una larga duración de la enfermedad, utilizando el procesamiento emocional como medida de cognición social.

\section{MÉTODO}

\section{Participantes}

En esta investigación participaron 60 personas con diagnóstico clínico de esquizofrenia según los criterios DSM-IV (American Psychiatric Association, 1994). Todos los participantes asistían a diversos Centros de Rehabilitación e Integración Social : 20 personas del Centro de Rehabilitación de Villena, 22 personas del Centro de Rehabilitación de Gandía La Safor, 15 personas del Centro de Rehabilitación de Valencia-Acova, y 3 personas del Centro de Rehabilitación de Valencia-Velluters. Todos ellos dieron su consentimiento informado para participar en este estudio. Los criterios de exclusión fueron: historia de daño neurológico, problemas de abuso de drogas o alcohol, problemas visuales o un C.I. estimado por debajo de 70 o superior a 115. Todos los participantes se encontraban clínicamente estables y estaban recibiendo tratamiento farmacológico y rehabilitación psicosocial durante el periodo de evaluación. Las características sociodemográficas se pueden ver en la Tabla 1. 
Tabla 1. Características de la Muestra $(N=60)$

\begin{tabular}{|c|c|}
\hline & $\mathrm{n}(\%)$ \\
\hline Hombres & $47(78.33)$ \\
\hline Mujeres & $13(21.67)$ \\
\hline Solteros & $56(93.33)$ \\
\hline Estado Civil & $3(5)$ \\
\hline Divorciados & $1(1.66)$ \\
\hline Trabaja & $2(3.51)$ \\
\hline $\begin{array}{r}\text { Trabajo de tipo } \\
\text { economía sumeroida }\end{array}$ & $4(7.02)$ \\
\hline No trabaja & $51(89.47)$ \\
\hline Paranoide & $33(55)$ \\
\hline Diagnóstico & $17(28.33)$ \\
\hline de Esquizofrenia & $7(11.67)$ \\
\hline Desorganizada & $1(1.66)$ \\
\hline Dual con otro trast. & $2(3.33)$ \\
\hline \multicolumn{2}{|c|}{ Media y DT } \\
\hline Edad & $39.70(8.42 \mathrm{DT})$ \\
\hline Coeficiente Intelectual estimado & $85.17(13.01 \mathrm{DT})$ \\
\hline Años de educación & $9.52(2.85 \mathrm{DT})$ \\
\hline Duración de la enfermedad (años) & $17.47(8,70 \mathrm{DT})$ \\
\hline $\begin{array}{l}\text { Asistencia al Centro de } \\
\text { Rehabilitación (años) }\end{array}$ & $3.42(2.61 \mathrm{DT})$ \\
\hline
\end{tabular}

$*_{\mathrm{n}}=57$

\section{Instrumentos de evaluación}

Se estimó el Coeficiente Intelectual con la versión abreviada, desarrollada por Blyler, Gold, Iannone y Buchanan (2000), de la Escala de Inteligencia para Adultos de Wechsler-III (WAIS-III) (Wechsler, 1999). Para evaluar el funcionamiento ejecutivo y la flexibilidad cognitiva se utilizó El Wisconsin Card Sorting Test (WCST) (Heaton, Chelune, Talley, Kay y Curtiss, 2001). De esta prueba se obtuvieron el número de categorías conseguidas y el número de errores de perseveración. La evaluación de la memoria cotidiana fue llevada a cabo con el Rivermead Behavioral Memory Test (RBMT) (Wilson, Cockburn y Baddeley, 1991). El RBMT está compuesto por 12 subtests que valoran el recuerdo de tareas cotidianas y la retención del tipo de información necesaria para un funcionamiento cotidiano adecuado. Concretamente se ha utilizado el subtest 6 (versión B) "recuerdo de la historia" que mide el recuerdo inmediato y el recuerdo diferido. Para evaluar la memoria de trabajo se utilizó el subtest de Dígitos 
del WAIS-III. La evaluación de la atención y vigilancia fuen realizada con las pruebas de Figuras Incompletas y de Dígitos del WAIS-III. Por último la prueba de Claves del WAIS-III se usó para evaluar la velocidad de procesamiento.

El Procesamiento Emocional se evaluó con la Face Emotion Identification Task (FEIT) (Kerr y Neale, 1993) y de la Face Emotion Discrimination Task (FEDT) (Kerr y Neale, 1993). La FEIT está compuesta por 19 fotografías de rostros en blanco y negro que expresan seis emociones básicas (alegría, tristeza, enfado, miedo, sorpresa y vergüenza). La persona debe identificar cuál de las seis emociones representa mejor el afecto expresado en el rostro de la persona que aparece en la fotografía. La FEDT está compuesta a su vez por 30 pares de fotografías que expresan las seis emociones básicas ya mencionadas, sin embargo en este instrumento se requiere que la persona determine si los dos rostros de personas diferentes presentados al mismo tiempo expresan la misma emoción o emociones diferentes. Ambas pruebas utilizan fotografías realizadas por Izard (1971) y Ekman y Friesen (1976). La puntuación total se obtiene sumando el número de respuestas correctas. La consistencia interna de la FEIT y la FEDT es de 0.59 y 0.70 respectivamente, siendo instrumentos avalados por su uso en otras investigaciones anteriores (Kerr y Neale, 1993; Mueser et al., 1996; Penn, Combs, Ritchie, Francis, Morris y Townsend, 2000).

En la evaluación del Funcionamiento Social se utilizó la adaptación al castellano de la escala Life Skills Profile (LSP) (Rosen, Hadzi-Pavlovic y Parker, 1989), "Perfil de Habilidades de la Vida Cotidiana" (Bulbena, Fernández de Larrinoa y Domínguez, 1992). La LSP consta de 39 ítems que son valorados por medio de una escala likert de 4 opciones de respuesta que se puntúan de 1 a 4 . La puntuación total en la LSP va desde 39 a 156. Puntuaciones altas indicarían un buen funcionamiento diario y puntuaciones bajas indicarían una disfunción significativa y déficits en las habilidades. Las subescalas de la versión española son 5: Autocuidado, Comportamiento Social Interpersonal, Comunicación y Contacto Social, Comportamiento Social No Personal y Vida Autónoma (Fernández de Larrinoa, Bulbena y Domínguez, 1992). Además, se utilizó versión autoinformada de la adaptación española de la Social Functioning Scale (SFS) (Birchwood, Smith, Cochrane, Wetton y Copetake, 1990), "Escala de Funcionamiento Social" (Vázquez y Jiménez, 2000). Este instrumento está compuesto por 79 ítems divididos en 7 subescalas: Aislamiento/Integración social, Comunicación Interpersonal, Autonomía-Ejecución, Autonomía-Competencia, Tiempo Libre, Actividades Prosociales, y Empleo/Ocupación. A mayor puntuación, mejor funcionamiento social global.

\section{RESULTADOS}

Los análisis de datos se centraron en el cálculo de las correlaciones (Coeficiente de correlación de Pearson) entre las puntuaciones obtenidas en todas las variables medidas. En la tabla 2 aparecen las puntuaciones obtenidas en las variables evaluadas. 
Tabla 2. Estadísticos Descriptivos

\begin{tabular}{|c|c|c|c|}
\hline & Min-Max. & Media & DT \\
\hline \multicolumn{4}{|l|}{ Medidas Neurocognitivas: } \\
\hline RBMT-RI* & $2-13$ & 7.00 & 2.61 \\
\hline RBMT-RD* & $0-13$ & 5.86 & 2.68 \\
\hline Claves WAIS-III & $1-12$ & 6.98 & 2.53 \\
\hline Digitos WAIS-III & $4-14$ & 8.22 & 2.60 \\
\hline Completación Figuras WAIS-III & $2-14$ & 8.20 & 2.11 \\
\hline CI WAIS-III & $70-115$ & 85.17 & 13.00 \\
\hline WCST-Cat & $0-6$ & 3.27 & 2.11 \\
\hline WCST-EP & $5-93$ & 33.80 & 20.87 \\
\hline \multicolumn{4}{|l|}{ Medidas de Procesamiento Emocional: } \\
\hline FEIT & $6-17$ & 11.98 & 2.92 \\
\hline FEDT & $17-29$ & 24.27 & 2.93 \\
\hline \multicolumn{4}{|l|}{ Medidas de Funcionamiento Social: } \\
\hline SFS total* & $\begin{array}{l}79.29- \\
118.14\end{array}$ & 99.33 & 8.95 \\
\hline F1-SFS: Aislamiento/Integración* & $72-127$ & 102.68 & 13,98 \\
\hline F2-SFS: Comunicación Interpersonal* & $58-119$ & 100.86 & 16,06 \\
\hline F3-SFS: Autonomía ejecución* & $73-123$ & 100.93 & 12,16 \\
\hline F4-SFS: Autonomía competencia* & $64-113$ & 96.60 & 11.90 \\
\hline F5-SFS: Ocio* & $84-135$ & 104.11 & 11.40 \\
\hline F6-SFS: Actividad Prosocial* & $80-119$ & 96.96 & 9.06 \\
\hline F7-SFS: Empleo* & $78-117$ & 93.19 & 14.36 \\
\hline LSP total & $89-153$ & 128.83 & 14.15 \\
\hline LSP-F1: Autocuidado* & $21-40$ & 34.56 & 4.36 \\
\hline LSP-F2: Comportamiento Social & $25-40$ & & \\
\hline Interpersonal* & & 34.74 & 4.26 \\
\hline LSP-F3: Comunicación-contacto social* & $11-24$ & 18.39 & 3.53 \\
\hline $\begin{array}{l}\text { LSP-F4: Comportamiento social no } \\
\text { personal* }\end{array}$ & $15-24$ & 22.02 & 2.10 \\
\hline LSP-F5: Vida Autónoma* & $7-28$ & 18.46 & 4.37 \\
\hline
\end{tabular}

$\mathrm{n}=60,{ }^{*} \mathrm{n}=57$. RBMT-RI, Rivermead recuerdo inmediato; RBMT-RD, Rivermead recuerdo diferido; WCST-Cat, categorías completadas en el Test de Clasificación de Tarjetas Wisconsin; WCSTEP, errores perseverativos en el Test de Clasificación de Tarjetas Wisconsin; SFS, Escala de Funcionamiento Social; LSP, Perfil de Habilidades de la Vida Cotidiana. 
En la tabla 3 aparecen las correlaciones entre las variables neurocognitivas y el procesamiento emocional. El rendimiento en el subtest de Recuerdo Inmediato del RBMT correlacionó significativamente con los rendimientos en las tareas de identificación y de discriminación de emociones. El rendimiento en el subtest de Recuerdo Diferido del RBMT también correlacionó significativamente con el rendimiento en la tarea de discriminación de emociones. Finalmente, el número de errores perseverativos (WCST) correlacionó significativamente con los resultados de la tarea de identificaciones de emociones.

\section{Tabla 3. Relaciones Entre Neurocognición y Procesamiento Emocional}

\begin{tabular}{|llc|}
\hline & FEIT & FEDT \\
RBMT-RI & $0.268^{*}$ & $0.342^{* *}$ \\
RBMT-RD & 0.189 & $0.317^{*}$ \\
Claves (WAIS-III) & -0.171 & 0.005 \\
Dígitos (WAIS-III) & 0.031 & 0.015 \\
Completación Figuras (WAIS-III) & 0.083 & 0.152 \\
WCST -Cat & 0.086 & 0.145 \\
WCST-EP & $-0.302^{*}$ & -0.098 \\
\hline
\end{tabular}

Correlaciones de Pearson, $\mathrm{n}=60,{ }^{*} \mathrm{p}<0.05 ; * * \mathrm{p}<0.01$.

FEIT, Facial Emotion Identification Task; FEDT, Facial Emotion Discrimination Task; RBMT-RI, Rivermead recuerdo inmediato; RBMT-RD, Rivermead recuerdo diferido; WCST-Cat, categorías completadas en el Test de Clasificación de Tarjetas Wisconsin; WCST-EP, errores perseverativos en el Test de Clasificación de tarjetas Wisconsin.

En la Tabla 4 aparecen los resultados del análisis correlacional entre la neurocognición y en funcionamiento social. El rendimiento en el recuerdo inmediato correlacionó de significativamente con la sub-escala de Comunicación Interpersonal de la SFS. El rendimiento en el sub-test de Dígitos del WAIS-III (memoria de trabajo / atención y concentración) correlacionó significativamente pero de forma negativa con la sub-escala de Autonomía Ejecución de la SFS. Las puntuaciones en el subtest de Completación de Figuras del WAIS-III también correlacionaron significativamente con los rendimientos en las sub-escalas de Autonomía Ejecución y Autonomía Competencia de la SFS. 
Tabla 4. Relaciones Entre Neurocognición y Funcionamiento Social

\begin{tabular}{|c|c|c|c|c|c|c|c|}
\hline & RBMT-RI & RBMT-RD & $\begin{array}{c}\text { Claves } \\
\text { (WAIS-III) } \\
\end{array}$ & $\begin{array}{c}\text { Dígitos } \\
\text { (WAIS-III) }\end{array}$ & $\begin{array}{l}\text { Comp.Figuras } \\
\text { (WAIS-III) }\end{array}$ & SWC & VCST-EP \\
\hline LSP total & -0.041 & 0.128 & 0.137 & 0.098 & 0.144 & $0.443 * *$ & $-0.319 *$ \\
\hline LSP-F1 & -0.127 & -0.012 & 0.014 & -0.046 & 0.071 & $0.281^{*}$ & -0.145 \\
\hline LSP-F2 & -0.099 & -0.099 & 0.145 & 0.221 & 0.172 & $0.339 * *$ & -0.177 \\
\hline LSP-F3 & 0.157 & 0.217 & 0.04 & 0.081 & -0.01 & $0.324 *$ & $-0.363 * *$ \\
\hline LSP-F4 & -0.033 & 0.026 & 0.185 & 0.151 & 0.072 & 0.156 & -0.032 \\
\hline LSP-F5 & -0.019 & 0.141 & 0.006 & -0.044 & 0.172 & $0.376 * *$ & $-0.307 *$ \\
\hline SFS total & 0.151 & 0.253 & 0.046 & -0.230 & 0.162 & 0.010 & -0.038 \\
\hline SFS-F1 & -0.018 & 0.054 & 0.003 & -0.080 & -0.057 & -0.176 & 0.170 \\
\hline SFS-F2 & 0.145 & $0.295^{*}$ & 0.077 & -0.174 & 0.012 & -0.148 & 0.118 \\
\hline SFS-F3 & 0.137 & 0.192 & 0.056 & $-0.278^{*}$ & $0.351 * *$ & 0.080 & -0.104 \\
\hline SFS-F4 & 0.231 & 0.243 & 0.199 & 0.078 & $0.297 *$ & 0.005 & -0.119 \\
\hline SFS-F5 & -0.022 & 0.054 & -0.012 & -0.256 & 0.163 & 0.034 & -0.037 \\
\hline SFS-F6 & 0.035 & 0.099 & -0.205 & -0.110 & -0.076 & -0.174 & 0.093 \\
\hline SFS-F7 & 0.201 & 0.250 & 0.038 & -0.160 & 0.123 & $0.391 * *$ & $-0.307 *$ \\
\hline
\end{tabular}

Correlaciones de Pearson, $\mathrm{n}=60,{ }^{*} \mathrm{p}<0.05 ;{ }^{*} \mathrm{p}<0.01$

RBMT-RI, Rivermead recuerdo inmediato; RBMT-RD, Rivermead recuerdo diferido; WCST-Cat, categorías completadas en el Test de Clasificación de Tarjetas Wisconsin; WCST-EP, errores perseverativos en el Test de Clasificación de Tarjetas Wisconsin; LSP total, puntuación total en el Perfil de Habilidades de la Vida Cotidiana; LSP-F1, subescala Autocuidado del LSP; LSP-F2, sub-escala Comportamiento Social Interpersonal del LSP; LSP-F3, sub-escala Comunicación-contacto social del LSP; LSP-F4, sub-escala Comportamiento Social no personal del LSP; LSP-F5, sub-escala vida autónoma del LSP; SFS total, puntuación total en la Escala de Funcionamiento Social; SFS-F1, Sub-escala Aislamiento/Integración de la SFS; SFS-F2, Sub-escala Comunicación Interpersonal de la SFS; SFS-F3, Sub-escala Autonomía Ejecución de la SFS; SFS-F4, sub-escala Autonomía-competencia de la SFS; SFS-F5, sub-escala Ocio de la SFS; SFS-F6, sub-escala Actividad Prosocial de la SFS; SFS-F7, sub-escala Empleo de la SFS.

El número de categorías completadas en el WCST, medida para evaluar la flexibilidad cognitiva y funcionamiento ejecutivo, correlacionó de forma significativa con el rendimiento global en el Perfil de Habilidades para la Vida Cotidiana (LSP) y sus sub-escalas de Autocuidado, Comportamiento Social Interpersonal, Comunicación y Contacto Social y Vida Autónoma. Además, la cantidad de errores perseverativos en el WCST correlacionó significativamente con el 
rendimiento global en el Perfil de Habilidades para la vida cotidiana (LSP) y con dos de sus sub-escalas: la sub-escala de Comunicación y Contacto Social y la su-escala de Vida Autónoma.

Finalmente, las dos medidas de la tarea de flexibilidad cognitiva y funcionamiento ejecutivo correlacionaron significativamente con el rendimiento en la sub-escala de Empleo de la SFS. El número de categorías completadas correlacionó de forma positiva y la cantidad de errores perseverativos de forma negativa.

Los resultados del cálculo de correlaciones entre procesamiento emocional y funcionamiento social aparecen en la tabla 5. El rendimiento en la tarea de identificación de emociones, únicamente correlacionó de forma significativa con el rendimiento en la sub-escala de Comunicación y Contacto Social del LSP. El rendimiento en la tarea de discriminación de emociones no correlacionó de forma significativa con ninguna de las áreas de funcionamiento social evaluadas en este estudio.

Tabla 5. Relaciones Entre Procesamiento Emocional y Funcionamiento Social

\begin{tabular}{|lcr|}
\hline & FEIT & FEDT \\
\hline LSP total & 0.027 & 0.083 \\
& & \\
LSP-F1 & -0.109 & 0.013 \\
LSP-F2 & -0.036 & 0.075 \\
LSP-F3 & $0.292 *$ & 0.257 \\
LSP-F4 & -0.231 & -0.064 \\
LSP-F5 & -0.108 & 0.073 \\
& & \\
SFS total & 0.050 & 0.040 \\
& & \\
SFS-F1 & 0.014 & -0.060 \\
SFS-F2 & 0.048 & 0.009 \\
SFS-F3 & -0.028 & 0.047 \\
SFS-F4 & 0.014 & 0.076 \\
SFS-F5 & -0.047 & -0.040 \\
SFS-F6 & 0.150 & -0.019 \\
SFS-F7 & 0.106 & 0.165 \\
\hline
\end{tabular}

Correlaciones de Pearson, $\mathrm{n}=57,{ }^{*} \mathrm{p}<0.05 ;{ }^{*} \mathrm{p}<0.01$. 
FEIT, Facial Emotion Identification Task; FEDT, Facial Emotion Discrimination Task; LSP total, puntuación total en el Perfil de Habilidades de la Vida Cotidiana; LSP-F1, subescala Autocuidado del LSP; LSP-F2, sub-escala Comportamiento Social Interpersonal del LSP; LSP-F3, sub-escala Comunicación-contacto social del LSP; LSP-F4, sub-escala Comportamiento Social no personal del LSP; LSP-F5, sub-escala vida autónoma del LSP; SFS total, puntuación total en la Escala de Funcionamiento Social; SFS-F1, Sub-escala Aislamiento/Integración de la SFS; SFS-F2, Sub-escala Comunicación Interpersonal de la SFS; SFS-F3, Sub-escala Autonomía Ejecución de la SFS; SFS-F4, sub-escala Autonomía-competencia de la SFS; SFS-F5, sub-escala Ocio de la SFS; SFS-F6, sub-escala Actividad Prosocial de la SFS; SFS-F7, sub-escala Empleo de la SFS.

\section{DISCUSIÓN}

Las medidas neurocognitivas que correlacionaron de forma significativa con las medidas del Procesamiento Emocional fueron el recuerdo inmediato y el diferido, el funcionamiento ejecutivo y la flexibilidad cognitiva. Estos datos indican de que una adecuada memoria de trabajo, flexibilidad y razonamiento cognitivo ayudan en tareas específicas de la cognición social como el identificar y discriminar las emociones.

Las medidas de evaluación neurocognitivas correlacionaron de forma positiva con más áreas del funcionamiento social que las dos medidas de cognición social. Este resultado ven en la misma línea que el presentado en el trabajo de Green et al. (2000), en el que se estima que la neurocognición explica entre un $20 \%$ y un $60 \%$ de la varianza en el rendimiento del funcionamiento social y con la numerosa evidencia que correlaciona los rendimientos en tareas neurocognitivas y en funcionamiento social (Addington, Mc Cleary y Munroe-Blum, 1998; Addington y Addington, 1999; Addington y Addington, 2000; Bellack, Gold y Buchanan, 1999; Green et al., 2000; McGurk y Mueser, 2003; Milev, Ho, Arndt y Andreasen, 2005).

Las medidas neurocognitivas obtenidas que han mostrado una relación significativa áreas del funcionamiento comunitario son: memoria inmediata o de trabajo, atención y concentración, funcionamiento ejecutivo y flexibilidad cognitiva. La atención es considerada predictora del resultado funcional en áreas como la competencia social y el funcionamiento comunitario (Addington et al., 1998). En el presente estudio un rendimiento alto en tareas que requieren el uso de la atención y concentración correlaciona con una alta capacidad y alto rendimiento en actividades diarias que implican autonomía e independencia. El rendimiento en la tarea de funcionamiento ejecutivo y flexibilidad cognitiva correlacionó de forma positiva con el rendimiento en una medida general de habilidad para la vida diaria y, en especial, con varias de sus sub-dimensiones, como el funcionamiento interpersonal, la comunicación y contacto social, y en especial con conductas de autocuidado y de independencia o autonomía. Rund y Borg (1999) indican también la importancia de la flexibilidad cognitiva y el funcionamiento 
ejecutivo y su relación con el funcionamiento ocupacional.

A diferencia de los hallazgos de diversos estudios que muestran a la cognición social como variable mediadora entre la neurocognición y el funcionamiento social (Brekke et al., 2005; Green y Nuechterlein, 1999; Vauth et al., 2004), en este estudio las medidas de reconocimiento de los afectos correlacionan débilmente con el funcionamiento social. Únicamente el rendimiento en la identificación de emociones correlaciona de forma positiva con la comunicación y contacto social. Este resultado indica que para comunicarse de forma adecuada y funcional son necesarios procesos de cognición social como el reconocimiento de la emoción en otras personas. El hecho no hallar más correlaciones entre cognición social y funcionamiento social, puede explicarse por haber utilizado instrumentos que evalúan sólo un área de este constructo, el procesamiento de la emoción. El estudio de Brüne (2005) tampoco halló una relación significativa entre la ejecución en el reconocimiento de los afectos y el funcionamiento social. Sin embargo Brüne (2005) encontró que los resultados en las pruebas de Teoría de la Mente fueron el predictor más significativo de los déficits en el comportamiento social.

Los hallazgos de cómo influye el rendimiento en el reconocimiento de los afectos sobre el funcionamiento social están asociados también a la amplitud del constructo de funcionamiento social y por ende al tipo de instrumento utilizado para evaluarlo. En este estudio se han utilizado dos medidas de evaluación de funcionamiento comunitario (una autoaplicada y otra heteroaplicada), sin embargo, no se evaluaron otras dimensiones del funcionamiento social como las habilidades sociales, la resolución de problemas sociales o el comportamiento en el entorno. Los resultados nos muestran que el reconocimiento de la emoción no predice el rendimiento general en las habilidades para la vida cotidiana pero sí hay evidencia de otros estudios en los que predice otras áreas del funcionamiento social como las habilidades sociales (Cohen, Forbes, Mann y Blanchard, 2006; Ihnen et al., 1998), el rendimiento en el trabajo (Kee et al., 2003) o las habilidades interpersonales (Pinkham y Penn, 2006; Poole et al., 2000).

Un meta-análisis reciente de la relación entre neurocognición, cognición social y funcionamiento social en esquizofrenia elaborado por Fett, Viechtbauer, Dominguez, Penn, van Os y Krabbendam (2011) concluye que la Teoría de la Mente es el área que ha demostrado una asociación significativa más fuerte con el funcionamiento comunitario y que el Procesamiento de la Emoción estaría más fuertemente asociado al comportamiento social en el entorno.

\section{AGRADECIMIENTOS}

Agradecemos la colaboración de los equipos de trabajo y de las personas que asisten a los Centros de Rehabilitación de: Villena, "La Safor" de Gandía y "Acova" y "Velluters" de Valencia. Este trabajo ha sido financiado por el Ministerio de Ciencia e Innovación, referencia del proyecto PSI2009-09421. 


\section{REFERENCIAS}

Addington, J. y Addington, D. (2000). Cognitive and social functioning in schizophenia: a 2.5 year follow-up study. Schizophrenia Research, 44, 47-56.

Addington, J. y Addington, D. (1999). Neurocognitive and social functioning in schizophrenia. Schizophrenia Bulletin, 25, 173-182.

Addington, J., Mc Cleary, L. y Munroe-Blum, H. (1998). Relationship between cognitive and social dysfunction in schizophrenia. Schizophrenia Research, 34, 59-66.

American Psychiatric Association (1994). Diagnostic and Statistical manual of Mental Disorders. Washington, DC: APA.

Barch, D. (2005). The relationships among cognition, motivation, and emotion in Schizophrenia: How much and how little we know. Schizophrenia Bulletin, 31, 875-881.

Bellack, A., Gold, J. y Buchanan, R. (1999). Cognitive Rehabilitation for schizophrenia: Problems, prospects, and strategies. Schizophrenia Bulletin, 25, 257-274.

Birchwood, M., Smith, J., Cochrane, R., Wetton, S. y Copestake, S. (1990). The Social Functioning Scale: The development and validation of a new scale of social adjustment for use in family intervention programmes with schizophrenic patients. British Journal of Psychiatry, 157, 853-859.

Blyler, C., Gold, J., Iannone, V. y Buchanan, R. (2000). Short form of the WAISIII for use with patients with schizophrenia. Schizophrenia Research, 46, 209-215.

Brekke, J., Kay, D., Lee, K. y Green, M. (2005). Biosocial pathways to functional outcome in schizophrenia: a path analytic model. Schizophrenia Research, $80,213-225$.

Brothers, L. (1990). The Social brain: A project for integrating primate behaviour and neurophysiology in new domain. Concepts in Neuroscience, 1, 27-61.

Brüne, M. (2005). Emotion recognition, 'theory of mind' and social behaviour in schizophrenia. Psychiatry Research, 133, 135-147.

Bryson, G., Bell, M., y Lysaker, P. (1997). Affect recognition in schizophrenia: a function of global impairment or a specific cognitive deficit. Psychiatry Research, 71, 105-113.

Bulbena, A., Fernández de Larrinoa, P., y Domínguez, A. (1992). Adaptación castellana de la escala LSP (Life Skills Profile) Perfil de Habilidades de la Vida Cotidiana. Estructura y Composición factorial. Actas Luso-Españolas de Neurología, Psiquiatría y Ciencias Afines. 20, 51-60.

Cohen, A., Forbes, C., Mann, M. y Blanchard, J. (2006). Specific cognitive deficits and differential domains of social functioning impairment in schizophrenia. Schizophrenia Research, 81, 227-238.

Ekman, P. y Friesen, W. (1976). Pictures of facial affect. Palo Alto, CA: Consulting Psychologists Press.

Fernández de Larrinoa, P., Bulbena, A. y Domínguez, A. (1992). Estudio de fia- 
bilidad, validez y consistencia interna de la escala LSP (Life Skills Profile). Perfil de Habilidades de la Vida Cotidiana. Actas Luso-Españolas de Neurología, Psiquiatría y Ciencias Afines, 20, 71-75.

Fett, A.K., Viechtbauer, W., Dominguez, M.G., Penn, D., van Os, J. y Krabbendam, L. (2011). The relationship between neurocognition and social cognition with functional outcomes in schizophrenia: A meta-analysis. Neuroscience and Biobehavioral Review, 35, 573-588.

Gold, J. y Harvey, P. (1993). Cognitive deficits in schizophrenia. Psychiatric Clinics of North America, 16, 295-312.

Green, M., Kern, R., Braff, D. y Mintz, J. (2000). Neurocognitive deficits and functional outcome in schizophrenia: are we measuring the "right stuff"? Schizophrenia Bulletin, 26, 119-136.

Green, M. y Nuechterlein, K. (1999). Should schizophrenia be treated as a neurocognitive disorder? Schizophrenia Bulletin, 25, 309-318.

Green, M. y Nuechterlein, K. (2004). The MATRICS initiative: Developing a consensus cognitive battery for clinical trials. Schizophrenia Research, 72, $1-3$.

Heaton, R., Chelune, C., Talley, J., Kay, G. y Curtiss, G. (2001) Test de Clasificación de Tarjetas de Wisconsin, WCST, Manual. Madrid: TEA Ediciones.

Hoff, A., Sakuma, M., Wieneke, M., Horon, R., Kushner, M. y De Lisi, L. (1999). Longitudinal Neuropsychological Follow - Up Study of Patients With FirstEpisode Schizophrenia. American Journal of Psychiatry, 156, 1336-1341.

Hooker, C. y Park, S. (2002) Emotion processing and its relationship to social functioning in schizophrenia patients. Psychiatry Research, 112, 41-50.

Ihnen, G., Penn, D., Corrigan, P. y Martin J. (1998). Social Perception and social skill in schizophrenia. Psychiatry Research, 80, 275-286.

Izard, C. (1971). The face of emotion. New York: Appleton-Century-Crofts.

Jaramillo, P., Fuentes, I. y Ruiz, J.C. (2009). Cognition, Social Cognition and Social Functioning in Schizophrenia. Psychology, Society \& Education, 1, 13-24.

Kee, K., Green, M., Mintz, J. y Brekke, J. (2003). Is emotion processing a predictor of functional outcome in schizophrenia? Schizophrenia Bulletin, 29, 487-497.

Kee, K., Kern, R. y Green, M. (1998). Perception of emotion and neurocognitive functioning in schizophrenia: what's the link? Psychiatry Research, 81, 57-65.

Kerr, S. y Neale, J. (1993). Emotion perception in schizophrenia: specific deficit or further evidence of generalized poor performance? Journal of Abnormal Psychology, 102, 312-318.

McGurk, S. y Mueser, K. (2003). Cognitive functioning and employment in severe mental illness. Journal of Nervous and Mental Disease, 191, 789-798.

Milev, P., Ho, B., Arndt, S. y Andreasen, N. (2005). Predictive values of neurocognition and negative symptoms on functional outcome in schizophrenia: a longitudinal first-episode study with 7-year follow -up. American Journal of 
Psychiatry, 162, 495-506.

Mueser, K., Doonan, R., Penn, D., Blanchard, J., Bellack, A., Nishith, P. y DeLeon, J. (1996). Emotion recognition and social competence in chronic schizophrenia. Journal of Abnormal Psychology, 105, 271-275.

Penn, D., Combs, D. y Mohamed, S. (2001). Social Cognition and Social Functioning in Schizophrenia. En P. Corrigan y D. Penn (Eds.), Social Cognition and Schizophrenia (97-121). Washington, DC: American Psychological Association.

Penn, D., Combs, D., Ritchie, M., Francis, J., Morris, S. y Townsend, M. (2000). Emotion recognition in schizophrenia: further investigation of generalized versus specific deficits models. Journal of Abnormal Psychology, 109, 512516.

Penn, D., Spaulding, W., Reed, D., y Sullivan, M. (1996). The relationship of social cognition to ward behavior in chronic schizophrenia. Schizophrenia Research, 20, 327-335.

Pinkham, A. y Penn, D. (2006). Neurocognitive and social cognitive predictors of interpersonal skill in schizophrenia. Psychiatry Research, 143, 167-178.

Poole, J., Tobias, F. y Vinogradov, S. (2000). The functional relevance of affect recognition errors in schizophrenia. Journal of the International Neuropsychological Society, 6, 649-658.

Rosen, A., Hadzi-Pavlovic, D., y Parker, G. (1989). The Life Skills Profile: A Measure Assessing Function and Disability in Schizophrenia. Schizophrenia Bulletin, 15, 325-337.

Rund, B. (1998). A review of longitudinal studies of cognitive functions in schizophrenia patients. Schizophrenia Bulletin, 24, 425-435.

Rund, B. y Borg, N. (1999). Cognitive deficits and cognitive training in schizophrenic patients: a review. Acta Psychiatrica Scandinavica, 100, 85-95.

Schneider, F., Gur, R.C., Gur, R.E. y Shtatsel, D. (1995). Emotional processing in schizophrenia: neurobehavioral probes in relation to psychopathology. Schizophrenia Research, 17, 67-75.

Schneider, F., Gur, R.C., Koch, K., Backes, V., Amunts, K., Shah, N.J., Bilker, W., Gur, R.E. y Habel, U. (2006). Impairment in the Specificity of emotion processing in Schizophrenia. American Journal Psychiatry, 163, 442-447.

Vauth, R., Rüsch, N., Wirtz, M. y Corrigan, P. (2004). Does social cognitive influence the relation between neurocognitive deficits and vocational functioning in schizophrenia? Psychiatry Research, 128, 155-165.

Vázquez, A. y Jiménez, R. (2000). Social Functioning Scale: new contributions concerning its psychometric characteristics in a Spanish adaptation. Psychiatry Research, 93, 247-256.

Wechsler, D. (1999). WAIS-III Escala de Inteligencia de Wechsler para Adultos IIII. Madrid: TEA Ediciones.

Wilson, B., Cockburn, J., Baddeley, A. y Hiorns, R. (1991). The Rivermead Behavioral Memory Test: supplement two. $2^{\mathrm{a}} \mathrm{ed}$. Bury St Edmunds: Thames Valley Test Company. 